\title{
*-INDUCTIVE LIMITS AND PARTITION OF UNITY
}

\author{
V. MURALI \\ Department of Mathematics \\ Rhodes University \\ Grahamstown 6140 \\ South Africa \\ (Received November 16, 1987 and in revised form September 22, 1988)
}

ABSTRACT In this note we define and discuss some properties of partition of unity on *-inductive limits of topological vector spaces. We prove that if a partition of unity exists on a *-inductive limit space of a collection of topological vector spaces, then it is isomorphic and homeomorphic to a subspace of a *-direct sum of topological vector spaces.

KEY WORDS AND PHRASES. Partition of unity, *-inductive limits, *-direct sum. AMS (Mos) SUBJECT CLASSIFICATION CODES. Primary: 46A12, 46A15.

Secondary: 46A99, 46M40.

\section{INTRODUCTION}

M. De Wilde [1] introduced the concept of partition of unity in an inductive limit space of a family of locally convex spaces which extends the usual partition of unity in function spaces. Around the same time S.O. Iyahen [2] introduced *-inductive limits of topological vector spaces, not necessarily locally convex, as a generalisation of inductive limits. In this paper, we consider the notion of partition of unity in *-inductive limit spaces of topological vector spaces and obtain some useful results some of which are analogous to De Wilde's results in [1]. In section 2, we briefly discuss the well-known concept of $\mathrm{F}-$ semi-norms in topological vector spaces. The details may be found in [6]. In section 3 , we define the concept of partition of unity in *-inductive limit and using this, obtain a family of $\mathrm{F}$-semi-norms defining the *-inductive limit topology. Finally we conclude with a representation theorem of *-inductive limit space with a partition of unity.

We prove that if a partition of unity exists on a *-inductive limit space of a collection of topological vector spaces, then it is isomorphic and homeomorphic to a subspace of a *-direct sum of topological vector spaces. 


\section{F-SEMI-NORMS}

Let $\mathrm{E}$ be a vector space over $\mathrm{k}$ where $\mathrm{k}$ is the field real or complex numbers.

DEFINITION 2.1

An $\mathrm{F} \rightarrow$ semi-norm on $\mathrm{E}$ is a mapping $\nu: \mathrm{E} \rightarrow \mathbb{R}$ such that

(ii) $\quad \quad \nu(\lambda \mathbf{x}) \leq \nu(\mathrm{x})$ for all $\mathrm{x} \in \mathrm{E}$ and for all $|\lambda| \leq 1$;

(iii) $\quad \quad \quad(\mathrm{x}+\mathrm{y}) \leq \nu(\mathrm{x})+\nu(\mathrm{y})$ for all $\mathrm{x}, \mathrm{y} \in \mathrm{E}$;

(iv) for each $x \in E, \nu(\lambda x) \rightarrow 0$ as $\lambda \rightarrow 0$.

Suppose that $\mathrm{V}=\left\{\nu_{\alpha}: \alpha \in \Lambda\right\}$ is a family of $\mathrm{F}$-semi-norms on $\mathrm{E}$. Then $\mathrm{V}$ determines a linear topology $\eta$ on E. A base of $\eta$-neighbourhoods of the origin in E consists of sets of the form

$$
\mathrm{U}_{\nu_{\alpha_{1}}{ }^{\prime} \nu_{\alpha_{2}}{ }^{\prime} \nu_{\alpha_{\mathrm{n}}}{ }^{\prime} \epsilon}=\left\{\mathrm{x} \in \mathrm{E}: \nu_{\alpha_{\mathrm{j}}}(\mathrm{x})<\epsilon, \mathrm{j}=1,2, \ldots, \mathrm{k}\right\}
$$

where $\epsilon$ is an arbitrary positive number and $\nu_{\alpha_{1}}, \nu_{\alpha_{2}}, \ldots, \nu_{\alpha_{n}}$ is any finite subcollection of V. Also, it is clear that each $\nu_{\alpha} \in \mathrm{V}$ is $\eta$-continuous and $\eta$ is the topology on $\mathrm{E}$ determined by the family $Q$ of all $\eta$-continuous $F$-semi-norms on $E$. In fact, an F-semi-norm $\mu \in Q$ if and only if, for each $\epsilon>0$ there exists a $\delta>0$ and a finite collection $\nu_{\alpha_{1}}, \nu_{\alpha_{2}}, \ldots, \nu_{\alpha_{\mathrm{n}}}$ of $\mathrm{V}$ such that

$$
\mathrm{U}_{\nu_{\alpha_{1}}, \nu} \nu_{\alpha_{2}}, \ldots, \nu_{\alpha_{\mathrm{n}}}, \delta \subseteq\{\mathrm{x}: \mu(\mathrm{x})<\epsilon\} .
$$

Conversely, we have the following:

$$
\text { THEOREM } 2.1
$$

A vector space topology on $\mathrm{E}$ can always be determined by a family of F-semi-norms. Proof: see [6], chapter 1, Proposition 2.

3. PARTITION OF UNITY:

Let $(E, \tau)$ be the *-inductive limit of a family of topological vector spaces $\left(E_{i}, \tau_{i}\right) i \in I$, an index family, relative to linear maps $u_{i}: E_{i} \rightarrow E$. Suppose further that the index set $I$ is directed and that for each pair indices $i, j \in I$ with $i<j$, there is a continuous linear map $v_{i j}: E_{i} \rightarrow E_{j}$ such that $u_{i}=u_{j} \circ v_{i j}$

DEFINITION 3.1 A partition of unity on $E$ is defined to be a family of linear maps $\left(T_{i}\right)(i \in I), T_{i}: E \rightarrow E_{i}$, which satisfies the following conditions.

$$
\begin{aligned}
& T_{i} \circ u_{j} \text { is continuous for each pair }(i-j) \text {. } \\
& \text { For each } j \in I, T_{i} u_{j}=0 \text { except for a finite number of } i \in I \text {. }
\end{aligned}
$$

Remark: We note that the condition (i) is equivalent to the following condition: (i) each $T_{i}: E \rightarrow E_{i}$ is continuous.

Example $\underline{3.2}$ Suppose $(\mathrm{E}, \tau)$ is the inductive limit of locally convex spaces $\left(\mathrm{E}_{\mathrm{i}}, \tau_{\mathrm{i}}\right)$ $(i \in I)$ with $\left\{\mathrm{T}_{\mathrm{i}}\right\}(\mathrm{i} \in \mathrm{I})$ is a partition of unity of $(\mathrm{E}, \tau)$. Then since $\tau$ is coarser than the 
*-inductive limit topology $\tau^{*}$ on $\mathrm{E}$, it follows that $\left\{\mathrm{T}_{\mathrm{i}}\right\}(\mathrm{i} \in \mathrm{I})$ is also a partition of unity of $\left(\mathrm{E}, \tau^{*}\right)$.

Example 3.3 Let $\left\{\mathrm{E}_{\mathrm{n}}\right\}(\mathrm{n}=1,2, \ldots)$ be a sequence of topological vector spaces, $\mathrm{E}$ be the *-direct sum of the $E_{n}$ 's as defined in [2], and let $\left\{P_{n}\right\}(n=1,2, \ldots)$ be the projection maps of $E$ onto $E_{n}$. Then, $E$ is the *-inductive limit of the sequence $\left.\underset{i=1}{\underset{i}{N}} E_{i}\right\}$ $(\mathrm{N}=1,2, \ldots)$ and the maps $\left\{\mathrm{P}_{\mathbf{n}}\right\}$ constitute a partition of unity.

We now consider some properties of the *-inductive limit space $(\mathrm{E}, \tau)$ with a partition of unity $\left\{\mathrm{T}_{\mathrm{i}}\right\}$ (i $\epsilon \mathrm{I}$ ) but first some notations.

For each $i \in I$, let $P_{i}$ be a family of $F$-semi-norms on $E_{i}$. Then $P_{i}$ determines a linear topology $\tau_{\mathrm{i}}$ on $\mathrm{E}_{\mathrm{i}}$ and let $\mathrm{Q}_{\mathrm{i}}=\left\{\mathrm{v}_{\mathrm{i}}^{\mathrm{a}}: \mathrm{a} \in \mathrm{\Gamma}_{\mathrm{i}}\right\}$ be the family of all $\tau_{\mathrm{i}}$-continuous $F$-semi-norms on $E_{i}$. For each collection $s$ of $F-$ semi-norms $\left\{v_{i}^{a}: v_{i}^{a} \in Q_{i}\right\}(i \in I)$ and each set $\sigma$ of positive real numbers $\left\{c_{i}\right\} i \in I$, we define a non-negative real-valued function $\pi_{\sigma}^{\mathrm{S}}$ on $\mathrm{E}$ by the equation

$$
\pi_{\sigma}^{\mathrm{s}}(\mathrm{x})=\sum_{\mathrm{i} \in \mathrm{I}} \mathrm{c}_{\mathrm{i}} \nu_{\mathrm{i}}^{\mathrm{a}}\left(\mathrm{T}_{\mathrm{i}} \mathrm{x}\right) \quad \text { for } \mathrm{x} \in \mathrm{E} .
$$

It is easy to verify that $\pi_{\sigma}^{\mathbf{S}}$ is a well-defined, F-semi-norm on E. By II we denote the family of all such $\mathrm{F}$-semi-norms $\pi_{\sigma}^{\mathrm{s}}$ for every collection of $\sigma$ and s.

THEOREM 3.4 The *-inductive limit topology $\tau$ on $\mathrm{E}$ is given by the family II of F-semi-norms $\pi_{\sigma}^{\mathrm{S}}$ defined by the equation 3.1 .

PROOF Let $\tau_{\text {II }}$ be the linear topology on E generated by the collection II We have to prove that $\tau=\tau_{\Pi}$. We will do this in two steps. First, to prove that $\tau_{\text {II }}$ is coarser than $\tau$, it is sufficient to show that each $\mathrm{u}_{\mathrm{j}}:\left(\mathrm{E}_{\mathrm{j}}, \tau_{\mathrm{j}}\right) \rightarrow\left(\mathrm{E}, \tau_{\mathbb{I I}}\right)$ is continuous. See [4]. Now each $u_{j}$ is continuous, if and only if for any $\pi_{\sigma} s \in \mathbb{I}, \pi_{\sigma^{0}{ }^{0} u_{j}}: E_{j} \rightarrow \mathbb{R}$ is continuous

In fact, for each $x \in E_{j}$,

$$
\pi_{\sigma}^{s}\left(u_{j} \mathrm{x}\right)=\sum_{i \in I} c_{i} \nu_{i}^{a}\left(T_{i} u_{j} \mathrm{x}\right) .
$$

But $T_{1} \circ u_{j}$ is equal to 0 except for a finite number of indices $i \in I$.

Let $\mathrm{J}=\left\{\mathrm{i} \in \mathrm{I}: \mathrm{T}_{\mathrm{i}} \mathrm{u}_{\mathrm{j}} \neq 0\right\}$.

Now each $\mathrm{T}_{\mathrm{i}} \mathrm{o} \mathrm{u}_{\mathrm{j}}$ is continuous from $\mathrm{E}_{\mathrm{j}}$ into $\mathrm{E}_{\mathrm{i}}$, and so $\nu_{\mathrm{i}}^{\mathrm{a}}\left(\mathrm{T}_{\mathrm{i}} \mathrm{o} \mathrm{u}_{\mathrm{j}}\right)$ is $\tau_{j}$-continuous Thus we can write $\pi_{\sigma^{\circ}}^{s} u_{j}=\sum_{i \in J} c_{i}\left(v_{i}^{a}\left(T_{i^{\circ}} u_{j}\right)\right)$ and so $\pi_{\sigma^{\circ}}^{s} u_{j}$ is continuous. From that 1 follows that $\tau_{\Pi I} \subseteq \tau$

$$
\begin{aligned}
& \text { For each } \mathrm{x} \in\left[\sum_{\mathrm{E}}^{\mathrm{E}}=\nu\left[\sum_{\mathrm{i} \in \mathrm{I}} \mathrm{u}_{10} \mathrm{~T}_{1} \mathrm{x}\right)\right] \\
& \leq \sum_{\mathrm{l} \in \mathrm{I}} \nu\left(\mathrm{u}_{\mathrm{i}} \mathrm{T}_{1} \mathrm{x}\right) .
\end{aligned}
$$


Now $\nu_{0} u_{i}$ is a $\tau_{i}$-continuous $F-$ semi-norm on $E_{j}$ and so belongs to $Q_{i}$. Hence

$$
\begin{aligned}
\nu(\mathrm{x}) & \leq \sum\left(\nu_{0} \mathrm{u}_{\mathrm{i}}\right)\left(\mathrm{T}_{\mathrm{i}} \mathrm{x}\right) \\
& =\pi_{\sigma}^{\mathrm{s}}(\mathrm{x}) .
\end{aligned}
$$

Here $s=\left\{\nu_{0} u_{i}\right\}(i \in I)$, and $c_{i}=1$ for each $i \in I$. This implies that the identity map (E, $\tau_{\text {II }} \rightarrow(\mathrm{E}, \tau)$ is continuous and so $\tau$ is coarser that $\tau_{\text {II }}$ as required. This completes the proof.

COROLLARY 3.5 If each $E_{i}(i \in I)$ is separated, then $(E, \tau)$ is separated.

THEOREM 3.6 If $B$ is a bounded set in $E$, then $T_{i} b=0$ except for a finite

number of indices $i \in I$. Hence $B$ is bounded in $E$ if and only if there exists a continuous linear mapping $\mathrm{T}$ from $\mathrm{E}$ onto some $\mathrm{E}_{\mathrm{i}}$ such that $\mathrm{B}=\mathrm{u}_{\mathrm{i}} \mathrm{TB}$.

The proof is analogous to that of the corresponding result in $([1], \mathrm{p} 3)$ and so is omitted here.

COROLLARY 3.7 If each $\left\{\mathrm{E}_{\mathrm{i}}\right\}$ is sequentially complete, then $\mathrm{E}$ is sequentially complete.

PROOF: Let $\left\{x_{n}\right\}$ be a Cauchy sequence in $E$. Then $\left\{x_{n}\right\}$ is a bounded set in $\mathrm{E}$, and so, by theorem 3.6, there exists a continuous linear mapping $\mathrm{T}$ from $\mathrm{E}$ into some $\mathrm{E}_{\mathrm{i}}$ such that $\left\{x_{n}\right\}=u_{i} T\left\{x_{n}\right\}$.

Since a continuous linear mapping from one topological vector space into another takes Cauchy sequences to Cauchy sequences, $T\left\{x_{n}\right\}$ is a Cauchy sequence in $E_{i}$. Now $E_{i}$ is sequentially complete, and so $T\left\{x_{n}\right\}$ converges to a point $x$ say in $E_{i}$. Therefore $u_{i} T\left\{x_{n}\right\}$ converges to $u_{i} x$, since $u_{i}$ is a continuous linear mapping. Therefore $\left\{x_{n}\right\}$ converges to a point in $\mathrm{E}$. Hence the result.

At present it is not known whether the completeness of each $\left(\mathrm{E}_{\mathrm{i}}, \tau_{\mathrm{i}}\right)$ implies the completeness of $\{\mathrm{E}, \tau)$. Lastly we prove that the collection of numbers in $\sigma$ of $\Pi_{\sigma}^{\mathrm{S}}$ can be chosen in an economical way. An useful application of this is given in [4].

PROPOSITION 3.8 Let $\sigma^{\prime}=\left\{c_{\mathrm{i}}: c_{\mathrm{i}} \geq 1\right\}$. If $\mathbb{I}^{\prime}$ denotes all $\mathrm{F}$-semi-norms of the form II $\sigma_{\sigma^{\prime}}^{\mathrm{S}}$ for various collections of $\mathrm{s}$ and $\sigma^{\prime}$, then $\tau_{\mathbb{I I}}=\tau_{\Pi^{\prime}}$, where $\tau_{\mathrm{II}}$ and $\tau_{\mathbb{I I}^{\prime}}$, denote the topology generated by II and $I^{\prime}$ respectively.

PROOF It is obvious that $\mathbb{I}^{\prime} \subseteq \mathbb{I}$ and so it is clear that $\tau_{\mathbb{I I}^{\prime}}$ is coarser $\operatorname{than} \tau_{\mathbb{I I}}$. Conversely let $\mathrm{U}$ be a $\tau_{\mathrm{II}}$-neighbourhood of the origin in $\mathrm{E}$. Then $\mathrm{V}$ contains a set $\mathrm{V}$ of the form

$$
\begin{aligned}
& \quad \mathrm{V}=\left\{\mathrm{x} \in \mathrm{E}: \pi_{\left.\sigma_{\mathrm{n}}{ }^{\mathrm{n}}(\mathrm{x})<\epsilon ; \mathrm{n}=1,2, \ldots \mathrm{m} ; \epsilon>0\right\} \text { where } \pi_{\sigma_{\mathrm{n}}}^{\mathrm{s}}(\mathrm{x})}\right. \\
& =\sum \mathrm{c}_{\mathrm{i}}{ }^{(\mathrm{n})} \nu_{\mathrm{i}}^{\mathrm{a}(\mathrm{n})}\left(\mathrm{T}_{\mathrm{i}} \mathrm{x}\right)
\end{aligned}
$$

Now let for any real number $r,[r]$ denote the greatest integer $\leq r$ then $c_{i}^{(n)}<\left[c_{i}^{(n)}\right]+1 ;$

and if we denote $\sigma_{n}^{\prime}=\left\{\left[c_{i}^{(n)}\right]+1\right\}$, then it is easy to see that $\pi_{\sigma_{n}^{\prime}}^{s}(x)$ for all $x \in E$. So we 
have $\mathrm{U} \supseteq \mathrm{V} \supseteq \mathrm{V}^{\prime}$, where $\mathrm{V}^{\prime}=\left\{\mathrm{x} \in \mathrm{E}: \boldsymbol{\pi}_{\sigma_{\mathrm{n}}^{\prime}}^{\mathrm{s}} \mathrm{n}(\mathrm{x})<\epsilon ; \mathrm{n}=1,2, \ldots, \mathrm{m}, \epsilon>0\right\}$ is a $\tau_{\Pi^{\prime}}$-neighbourhood of the origin. Thus we have $\tau_{\Pi}$ is coarser than $\tau_{\Pi^{\prime}}$, and so $\tau_{\mathbb{I I}}=\tau_{\Pi^{\prime}}$. 4. DIRECT SUM

In this section we give an analogue of a representation theorem given by D. Keim in [3]. Let $(\mathrm{E}, \tau)$ be the ${ }^{*}$-inductive limit of topological vector spaces $\left(\mathrm{E}_{\mathrm{i}}, \tau_{\mathrm{i}}\right)(\mathrm{i} \in \mathrm{I})$ relative to linear maps $u_{i}=E_{i} \rightarrow E$. Suppose, further that, a partition of unity $\left\{T_{i}\right\}$ is defined on $(E, \tau)$. Then we have the following representation theorem.

THEOREM 4.1 (E, $\tau)$ is isomorphic and homeomorphic to a subspace of a *-direct sum of topological vector spaces.

PROOF: Define a linear map from $(\mathrm{E}, \tau)$ into the *-direct sum of $\mathrm{E}_{\mathrm{i}}^{\prime} \mathrm{s}$ as follows:

$$
\text { : } \mathrm{E} \rightarrow \sum_{\mathrm{i} \in \mathrm{I}} \mathrm{E}_{\mathrm{i}} \text { given by }(\mathrm{x})=\left(\mathrm{T}_{\mathrm{i}} \mathrm{x}\right) \text { for } \mathrm{x} \in \mathrm{E} \text {. }
$$

This mapping is well-defined and one-to-one since $\left\{\mathrm{T}_{\mathrm{i}}\right\}$ satisfies the conditions (ii) and (iii) of partition of unity respectively. It is easy to check that is a linear map and so, is an isomorphism. Moreover that is continuous is shown as follows.

By condition (ii) of partition of unity, $\mathrm{T}_{\mathrm{i}} \mathrm{o} \mathrm{u}_{\mathrm{j}}=0$ except for a finite number of $\mathrm{i} \in \mathrm{I}$ and for each fixed $j \in I$. Let $i_{1}, i_{2}, \ldots, i_{n}$ be the finite number of indices such that $T_{i_{k}}{ }^{o}{ }_{j}=0$ for $k=1,2, \ldots, n$. Then ${ }_{o} u_{j}=\left(\sum I_{i_{k}}{ }_{0} T_{i_{k}}\right)_{0} u_{j}$ where $I_{i_{k}}$ is the injection map of $E_{i_{k}} \rightarrow \sum_{i \in I} E_{i}$. Now for each $i_{k}, k=1,2, \ldots, m, I_{i_{k}} \circ T_{i_{k}} \circ u_{j}$ is continuous by condition (i) of partition of unity and continuity of each $\mathrm{I}_{\mathrm{i}_{\mathrm{k}}}, \mathbf{k}=1,2, \ldots, \mathrm{n}$. Therefore ${ }_{0} \mathrm{u}_{\mathrm{j}}$ is continuous for each $\mathrm{j} \in \mathrm{I}$. Consequently is continuous [5], as required.

Conversely, let be a linear map defined by $: \sum_{i \in I} E_{i}+E$

$$
\Phi^{\prime}\left(\mathrm{x}_{\mathrm{i}}\right)=\sum_{\mathrm{i} \in \mathrm{I}} \mathrm{u}_{\mathrm{i}}\left(\mathrm{x}_{\mathrm{i}}\right) \text {. }
$$

This is well-defined since $x_{i}=0$ except for a finite number of $i \in I$. Moreover, is linear and $\Phi^{\prime} \mid(E)=\Phi^{-1}$. Also, $\Phi^{\prime}{ }_{0} I_{i}=u_{i}$ is continuous from $E_{j} \rightarrow E$ for each

$\mathrm{j} \in \mathrm{I}$. Hence is continuous. Thus an isomorphism and a homeomorphism onto $\sum_{i \in I} E_{i}$.

\section{REFERENCES}

1. DE WILDE, M., Inductive limits and partition of unity, Manuscripta Math.,5, (1971), 45-58.

2. IYAHEN, S.O., On certain classes of linear topological spaces, Proc. London Math. Soc. (3), 18, (1968), 285-307. 
3. KEIM, D. Induktive and projective limiten Mit Zerlegung der Einheit, Manuscripta Math., 10, (1973), 191-195.

4. MURALI, V. Partition of unity on algebraic inductive limits, Math. Japonica 22, (1977), 497-500.

5. MURALI, V. Suprabarrels in topological vector spaces, Math. Japonica 32, (1987) 797-800.

6. WEALBROECK, L. Topological Vector Spaces and Algebras, Springer-Verlag, Lecture notes (1971) 230. 


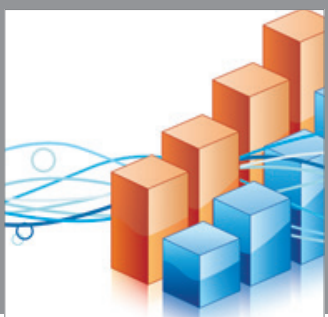

Advances in

Operations Research

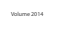

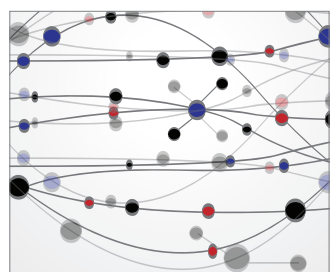

\section{The Scientific} World Journal
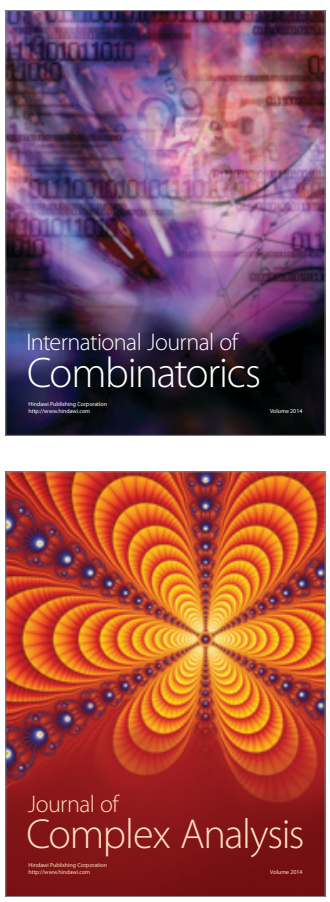

International Journal of

Mathematics and

Mathematical

Sciences
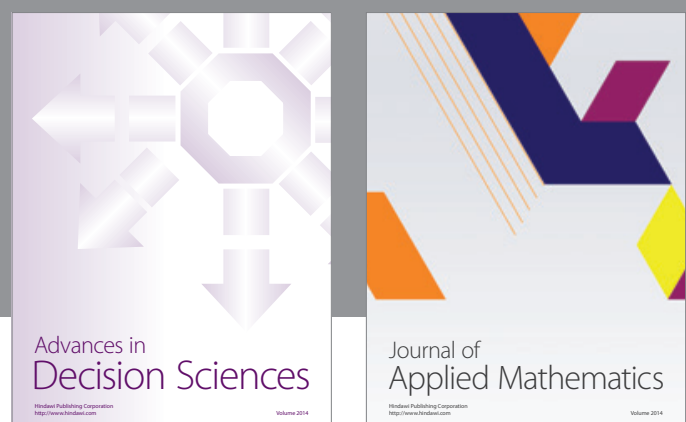

Journal of

Applied Mathematics
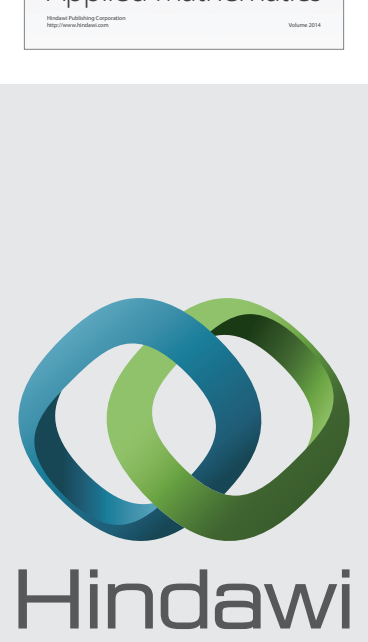

Submit your manuscripts at http://www.hindawi.com
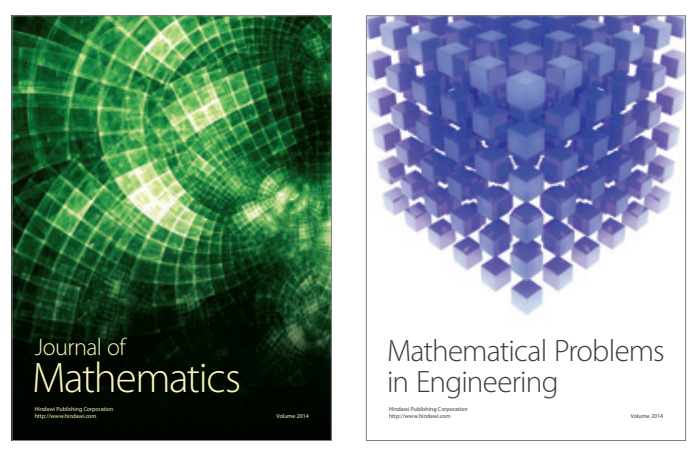

Mathematical Problems in Engineering
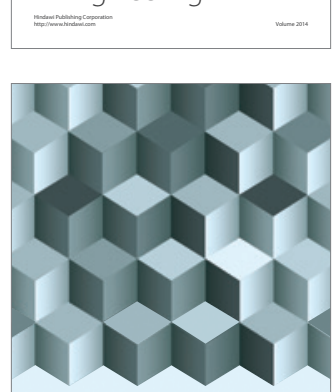

Journal of

Function Spaces
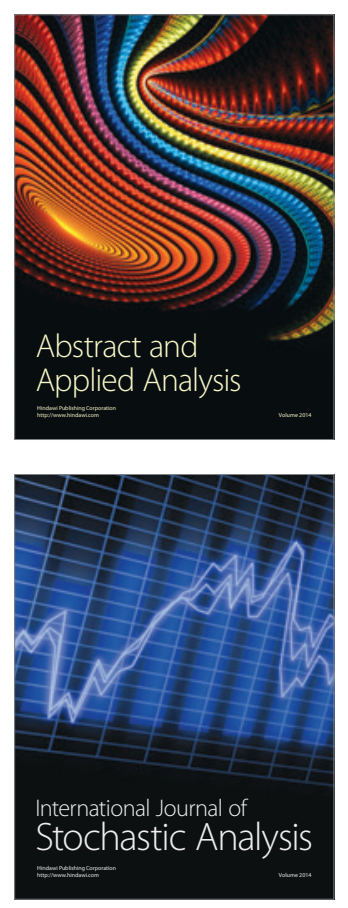

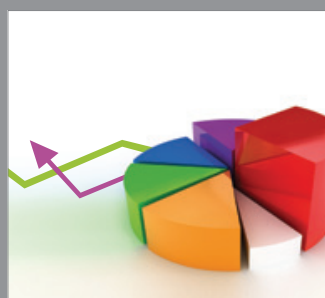

ournal of

Probability and Statistics

Promensencen
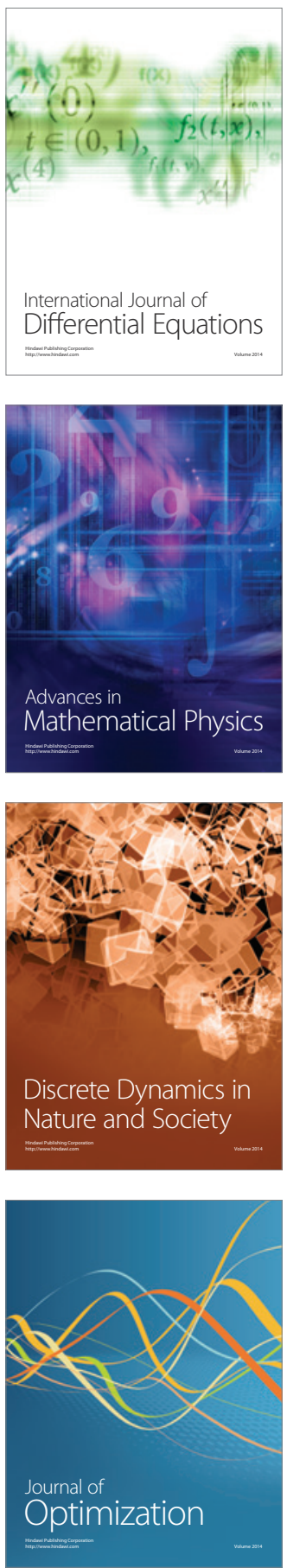